\title{
CASE OF REPEATED TUBAL PREGNANCY, DIAGNOSED ON EACH OCCASION BEFORE THE END OF THE FIRST MONTH. RE- PEATED ABDOMINAL SECTION. RECOVERY.
}

\author{
By E. RUMLEY DAWSON, L.R.C.P. (Lond.), M.R.C.S. (Eng.).
}

Cases of repeated tubal gestation are still of sufficient rarity to justify being recorded. The following case is so instructive that no apology is necessary for its somewhat detailed description :-

The patient, a healthy woman, of 25, was confined in July, 1890; the child, a girl, presented by the breech, and was stillborn. In July, 1893, the patient, owing to leaving off her undervest, took cold, and developed a sharp attack of general peritonitis; she was ill for four weeks. She married in 1894. Menstruation continued quite regularly; she remained absolutely sterile for seven years, no attempt being made to prevent conception. On June 12th, 1901, the day after her June period (a normal one both as to time and amount), coitus took place. The following, or July, period, due July 5th, did not appear, and she deemed herself pregnant. On July 9 th she first had acute abdominal pain, i.e., 27 days after the first coitus after the previous menstrual period. Irregular pains followed, and she noticed on the 10th and IIth pains shooting down the vagina and rectum, with a feeling of forcible distension in both. Repeated attacks of abdominal pain and faintness led to my being called to ser on July 12th, and on the 13th July, on vaginal examination, I diagnosed an early left tubal pregnancy, only 31 days having then elapsed since her previous normal menstrual period. Two days later, on July 15th, Mr. Bland-Sutton saw the patient, and confirmed the diagnosis, and on July $19 t h, 1901$, in my presence, he removed the left pregnant tube and ovary, in which was a well-marked corpus-luteum; typical villi being found at the placental site. At the operation it was noted, when an attempt was made to examine the right tube and right ovary, that these right appendages were so matted and bound down that Mr. Bland-Sutton could not bring them up to the abdominal incision for inspection. No attempt was made to liberate them. Subsequently menstruation continued quite regularly, but rather than run the risk of a subsequent pregnancy a check sponge was regularly introduced before coitus. The January, 1903, menstrual period was quite normal, both in amount and time. On Sunday, February 22nd, 1903, coitus took place, a sponge having previously been introduced; the period was due on the following Thursday (26th), but did not appear. Four days later, on Monday, March 2nd, irregular hæmorrhages began, and shooting pains down the vagina and rectum with much flatulence and feeling of distension in the latter. Next day there were irregular abdominal pains on the right side. On March 5th I was again sent for, and on vaginal examination I detected a small right tubal gestation, i.e., eleven days after the suspected insemination. Very sharp pains and faintness occurred during the night, after the raginal examination. Mr. Bland-Sutton saw the patient on March 8th, and once more confirmed the diagnosis, the tumour having much increased in size by this time. Next day, on March 9th, 1903, in my presence, he once more opened 
the abdomen and removed a small right tubal gestation; the accompanying ovary contained the corpus luteum. Only twenty months had therefore elapsed between the two operations. Mr. Bland-Sutton experienced no difficulty in now drawing the distended right tube and right ovary up to the incision, no adhesions being met with. A small mole was detected in the tube, and typical villi were afterwards found by the microscope. The patient, now that she has had both her ovaries removed, has been extremely troubled by hot flushes, and breaks out into profuse universal sweats. Menstruation, absolutely regular before the second operation, has completely stopped.

This case, apart from its initial rarity as an indubitable case of repeated tubal pregnancy, exemplifies the following points :-

1st. Complete removal of all ovarian tissue completely arrests menstruation.

2nd. It tends very greatly to support the theory that the chief cause of tubal pregnancy is a previous salpingitis. The attack of general peritonitis which the patient had ten years ago evidently so affected her tubes that for seven years she was sterile; then, probably as a result of the absorption of adhesions round the left tube, that tube became pregnant in 1901, and was removed. The right tube was at that time seen to be fixed and bound down.

Hahn I has lately gone so far as to state that "undoubtedly gonorrhcea is the most frequent cause of extra-uterine gestation." This still further supports Tait's theory that a previous salpingitis, however caused, is a predisposing cause of tubal pregnancy. From this case the fact that in one woman both tubes should become at different times pregnant, points to the conclusion that the tubes must have beer at fault. If a healthy tube were more likely to become pregnant than an unhealthy one, then it follows that most healthy women should have tubal pregnancies instead of uterine pregnancies. Certainly in this case an attack of peritonitis was followed by sterility, and, as in so many recorded instances, this long period of sterility was followed by her first tubal pregnancy; on the other hand, previously to the attack of peritonitis, she had a normal uterine pregnancy. It is significant that the tube of the opposite side has been often seen to be diseased by, among others, Strassmann ${ }^{2}$ and Haig Ferguson ${ }^{3}$ when operating for tubal pregnancies.

3rd. 'This case also exemplifies the fact that attempts to prevent pregnancy not only often fail to prevent the uterine implantation of the ovum, but similarly fail to prevent extra-uterine pregnancy. It certainly does not support the idea that artificial prevention of conception is a cause of tubal pregnancy, because no such attempts were being made when she became pregnant in her tube on the first occasion. 
I am also aware of a case where prolonged suckling, so often indulged in with the hope of preventing pregnancy, failed to prevent conception, fertilisation leading to a tubal pregnancy, and death, before the previous uterine child had been weaned.

Haig Ferguson ${ }^{4}$ mentions a case where " the patient lactated for 17 months in the hope of preventing another pregnancy, and yet in the irony of fate conception occurred in each tube."

4th. Absorption of inflammatory adhesions. Cases to absolutely prove absorption of adhesions are rare, and I cannot do better than quote Dr. Herman, ${ }^{5}$ who reports a definite case, though the interval between the two inspections of the tube in his case was over four years. He says :- " On October 26, 1886, the right ovary and tube were pulled up and removed in the usual way. Search was then made for the left ovary, but it was so embedded in adhesions that $I$ was quite unable to identify it. On February 20, 1901, I re-opened the abdomen. The left ovary and tube were only slightly adherent. The ovary and tube were removed." Further on he remarks :- "The absorption of peritoneal adhesions. Here the fact illustrated is one about which there can be no doubt. But cases proving it are exceptional. The ovary and tube, which were in 1886 so embedded in adhesions that the operator could not identify them, were in 1901 almost free, and were easily pulled up."

In the present case the absorption of adhesions is surmised to have occurred round the left tube, but is definitely proved to have happened in the case of the right tube. Only twenty months-July, 1901, to March, 1903-was sufficient to set the right tube quite free again after it was ascertained to be fixed. This liberation of the tube from its fixed position led to its becoming pregnant. After the removal of the left tube, the movements of the uterus in the acts of intercourse must have dragged to a greater extent upon, and thus stretched, the adhesions holding down the right tube, than when the left tube was in situ. There is no doubt but that the uterus is both straightened as well as drawn down by the round ligaments towards the vaginal outlet during intercourse, and the repetition of this movement must have been partly instrumental in freeing the right tube from its adhesions.

5th. Is the presence of pains shooting down the vagina and rectum, with tenesmus and a feeling of distension in both passages, a symptom of early tubal pregnancy? From an experience of five cases in general practice I think it must be. The importance of having some definite symptom to teach to the general practitioner as being indicative of tubal pregnancy renders it advisable to more 


\section{4 Journal of Obstetrics and Gynæcology}

closely inquire for this clinical symptom in all cases of extra-uterine pregnancy. The tendency, however, nowadays is to operate and give a pathological report, rather than to operate and give the clinical history of the case. In the Obstetrical Society's Transactions ${ }^{6}$ I published a case of fatal rupture of a very early tubal pregnancy, in which the symptoms of vaginal and rectal tenesmus, and pains and distension were so marked as to impress themselves very forcibly upon me. Later I ascertained the presence of identical symptoms in two other cases of extra-uterine pregnancy in the practices of local colleagues. When, therefore, in the present case the patient complained on both occasions of these symptoms I at once suspected, and then diagnosed the tubal pregnancy on each occasion. I know of no book which alludes to these symptoms, and many of the most recent writers on the subject do not mention them as suggestive symptoms, but speak only of indefinite pelvic pains with hæmorrhages and pieces of membrane as essentials to a correct diagnosis. There are, however, a few cases on record where in the clinical history these symptoms are casually mentioned, thus:--

M. F. Wagstaff, 7 reporting a case of extra-uterine foetation, says : "On April 28, 1829, I was desired to visit Mrs. Rondeau. She was suffering from constant tenesmus both of rectum and bladder."

Hasting Gilford ${ }^{8}$ : "The accident occurred in this case apparently as the result of straining at stool, but it is quite possible that a slight rupture had really taken place before, and was the cause of the desire to defæcate, for the patient did not actually get relief. That this is probably the true explanation is suggested by the report which I have seen of another case of rupture of later date, in which the first symptom was the sensation of the pressure of a hard substance obstructing the rectum and causing a desire to defecate."

Dr. Lewers, ${ }^{9}$ in reporting a case of repeated ectopic gestation, casually says:- " The pain is described as being a continuous aching in both groins, extending to the rectum and the vagina."

It therefore appears that these symptoms are present in at least some of the cases of extra-uterine gestation, and if further inquiry shows them to be usually present it should help to make the diagnosis of early tubal pregnancy easier, though it will probably always be tilue, as Dr. J. Baldwin says, " there are no pathognomonic symptoms of tubal or any other form of ectopic pregnancy." 
Rumley Dawson: Repeated Tubal Pregnancy 305

\section{References.}

1 Harrs. Quoted in British Medical Journal (Epitome), June 20, 1903.

2 Strassmans. Ibid, July 25, 1903.

3 Ferguson (HaIG). Journal of Obstets. and Gynac. Vol. iii., p. 326.

4 Journal of Obstetrics and Gynocology. Vol. iii., p. 327.

5 HeRman. Journal of Obstetrics and Gynocology. Vol. ii., pp. 226-228.

6 Trans. Obstet. Soc., London, 1898. Vol. xl., p. 155.

7 Wagstafr. The Medico-Chirurgical Review, 1831. Vol. xv., p. 187.

8 Gilford (H.). The Lancet, Feb. 20, 1897.

9 LEwERs. Transactions Obstetrical Soc., London, 1900 . Vol. xlii., p. 326. 